\title{
Das kolumbianische Verfassungsgericht und die sozialen Rechte
}

\author{
Von Rodolfo Arango, Bogotà*
}

\section{Einführung}

1991 hat eine verfassungsgebende Versammlung mit siebzig vom Volk direkt gewählten Mitgliedern eine neue und bedeutungsvolle Verfassung in Kolumbien verabschiedet. Dieser Aufsatz schildert die Rolle des kolumbianischen Verfassungsgerichts bei der Realisierung der sozialen Grundrechte. Er ist dazu in drei Abschnitte geteilt: Zuerst geht es um die geschichtlichen Umstände, die ermöglicht haben, eine so fortgeschrittene Verfassung mit umfangreichem Grundrechtskatalog und Grundrechtsschutzsystem zu schaffen. Danach werden kurz die kolumbianische Verfassungsgerichtsbarkeit, bzw. ihre Zusammensetzung, ihre Funktionen und ihr Einfluss beschrieben. Zuletzt sollen die Leitlinien der verfassungsrechtlichen Rechtsprechung zu sozialen Grundrechten dargestellt werden. Der vorliegende Beitrag soll die Reflektion über die politische Wirkung eines verfassungsrechtlichen Engagements für soziale Rechte aus der Perspektive einer der so genannten ,nicht wohl geordneten Gesellschaften“ ermöglichen ${ }^{1}$.

Um die Stellung des kolumbianischen Verfassungsgerichts zu sozialen Grundrechten zu verstehen, bedarf es einiger Daten: Kolumbien ist ca. dreimal so groß wie Deutschland, hat aber mit 44 Mio Einwohnern nur die Hälfte seiner Bevölkerung. Indigene Völker bilden 2\% der Gesamtbevölkerung. Kolumbien exportiert außer Rohstoffen und Agrarprodukten (hauptsächlich Kaffee, Bananen und Blumen, aber auch Kokain) Manufakturprodukte wie Textilien und Kunsthandwerk in die USA, nach Europa und neuerdings nach Venezuela. Mehr als die Hälfte der Bevölkerung Kolumbiens lebt unter der Armutsgrenze von 1 USDollar pro Kopf am Tag, auf dem Land sind es $80 \%$. Kolumbien ist nach Brasilien das Land Lateinamerikas mit der größten wirtschaftlichen Einkommensungleichheit. Sozial versichert sind nur ca. 55\% der Bevölkerung. Das Jahres-Durchschnittseinkommen pro Kopf liegt bei 4.000,- USD, ein Zehntel im Vergleich zu Deutschland. Kolumbien ist seit seiner Unabhängigkeit von Spanien (1819) eine demokratische Republik mit regelmäßigen Wahlen. Die politische Struktur besteht aus einem zentralistischem Präsidialsystem, das durch neuerliche Verfassungsreformen zur Ermöglichung der Präsidentenwiederwahl zulasten des föderativen und demokratischen Parteisystems geprägt ist.

Rodolfo Arango, Dr. jur. (Kiel), Ex-Magistrado Auxiliar de la Corte Constitucional; Profesor assistente de la Facultad de Derecho y Ciencias Polìticas y Sociales, Universidad de los Andes, Bogotá. E-Mail: rarango@uniandes.edu.co.

1

Rodolfo Arango, Der Begriff der sozialen Grundrechte, Baden-Baden 2001, p. 127. 


\section{A. Geschichtliche Umstände der Verfassungsänderung 1991}

\section{Auf internationaler Ebene}

Peter Häberle hat die Lage der Konstitutionalisierung der Weltgemeinschaft vor einigen Jahren mit Hilfe der Metapher der drei Wellen beschrieben. Die erste Welle betraf den Einfluss des amerikanischen Konstitutionalismus auf Deutschland und Japan nach dem zweiten Weltkrieg. Die zweite Welle erstreckte sich von Deutschland nach Spanien, Portugal und Asien. Die dritte Welle erfasste Lateinamerika und die ehemaligen Ostblockstaaten gegen Ende des vorigen Jahrhunderts. Die Annahme geschriebener Verfassungen mit umfangreichen Grundrechtskatalogen und Verfassungsgerichtsbarkeit ebenso wie eine an freiheitlichen und sozialen Grundprinzipien orientierte Grundrechtsauslegung, charakterisieren den so genannten Neukonstitutionalismus, der sich als vielversprechende politische Formel in Lateinamerika ausbreitete.

\section{Auf nationaler Ebene}

Auf nationaler Ebene bestimmten mehrere Faktoren den Verfassungsänderungsprozess. In den fünfziger Jahren dominierte die Gewalt zwischen den politischen Parteien. Der Ausgang dieser "Violencia" war ein Nationalpakt, der von 1958 bis 1974 die politische Macht paritätisch zwischen der liberalen und der konservativen Partei teilte. Diese Lösung schloss allerdings andere politische Gruppierungen von der Teilnahme am politischen Prozess aus. Kolumbien lebte mehr als dreißig Jahre in einem ständigen Ausnahmezustand mit einer zunehmenden Machtkonzentration weg von der Legislative hin zur Exekutive. Diese ständig angespannte Lage eskalierte mit der Gegenrepression der Guerilla und kulminierte im November 1985 in der blutigen Rückeroberung des von der M-19-guerilla besetzten Justizpalastes, wobei unter den mehr als 200 Todesopfern auch zwölf zuvor als Geiseln exekutierte Mitglieder des Obersten Gerichtshofs waren. Die Ermordung eines sehr populären Präsidentschaftskandidaten ein Jahr später und der Bombenterror der Drogenkartelle ließen die landesweiten Forderungen nach einer Verfassungsreform als Rechtsrahmen für einen grundlegenden Wandel nicht mehr verstummen.

\section{B. Das kolumbianische Verfassungsgericht}

Nach sechsmonatigen Beratungen verabschiedete die verfassungsgebende Versammlung in Juli 1991 die neue Verfassung. Dabei hatten das deutsche und das spanische Verfassungsrecht großen Einfluss. Einige der wichtigsten Verfassungsänderungen waren die Aufnahme einer umfangreichen Grundrechtecharta und die ebenfalls anspruchsvolle Errichtung einer eigenständigen Verfassungsgerichtsbarkeit. Das ging zulasten des Obersten Gerichtshofs (Corte Suprema de Justicia), der z.B. seine Kompetenz für die abstrakte Normenkontrolle an das Verfassungsgericht verlor. 


\section{Die Verfassungsgerichtsbarkeit}

Merkmale der kolumbianischen Verfassungskontrolle seit 1991 sind eine konzentrierte oder zentralisierte Verfassungsgerichtsbarkeit zur abstrakten Normenkontrolle und eine diffuse oder dezentralisierte Verfassungsgerichtsbarkeit zum Schutz der Grundrechte.

\section{Konzentrierte Verfassungsgerichtsbarkeit bei abstrakter Normenkontrolle}

An der Spitze der konzentrierten Verfassungsgerichtsbarkeit steht das kolumbianische Verfassungsgericht (Corte Constitucional). Das Plenum des Verfassungsgerichts entscheidet nach einem öffentlichen und partizipativen Verfahren über die Verfassungskonformität von einfachen Gesetzen. Die Entscheidungen des Verfassungsgerichts betreffen Verfassungsklagen, die seit 1991 von jedermann gegen Normen auf Gesetzesstufe erhoben werden können oder die automatische Verfassungskontrolle von Normen, die im Rahmen von Ausnahmezuständen erlassen werden. Außerdem überprüft das Verfassungsgericht die Verfassungsmäßigkeit von Verfassungsänderungsprojekten und internationalen Abkommen, jeweils aber nur bezüglich der Einhaltung einschlägiger Prozeduren (formale Verfassungsprüfung).

\section{Diffuse Verfassungsgerichtsbarkeit zum Grundrechtsschutz}

Zum Schutz der Grundrechte - die vor 1991 allenfalls auf dem Papier standen - hat die Verfassung von 1991 eine anspruchsvolle Neuerung getroffen: Alle Richter (d.h. Zivil-, Arbeits-, Straf-, Verwaltungsrichter) fungieren, wenn es um den Schutz der Grundrechte geht, als Verfassungsrichter. Jede Person, die sich in ihren Grundrechten verletzt fühlt, durch das Tun oder das Unterlassen von staatlichen Behörden und solchen Privaten, welche soziale Macht ausüben (Drittwirkung der Grundrechte), kann eine Verfassungsklage (acción de tutela) vor den am ortsnächsten amtierenden Richter erheben, die in maximal zehn Tagen entschieden sein muss. Dadurch kann der Richter die Verfassung direkt anwenden und verschiedene Anweisungen erteilen, damit der verfassungswidrige Akt außer Kraft gesetzt wird oder das verfassungswidrige Unterlassen endet. Die richterlichen Grundrechtsentscheidungen können angefochten werden vor dem Verfassungsgericht, das für eine einheitliche Rechtsprechung in Grundrechtsfragen zu sorgen hat. Nicht nur Abwehr-, sondern auch Organisations- und Leistungsrechte kommen in Betracht. Dabei wird auf die sozialen Grundrechte gesondert zurückzukommen sein.

Der Vorteil der diffusen Verfassungsgerichtsbarkeit gegenüber einem konzentrierten System, wie es Deutschland und Spanien kennen, liegt darin, dass die Rechtsuchenden nicht lange bis zur Entscheidung warten müssen. Seit 1992 sind bis Juli 2008 insgesamt ca. 16.000 Entscheidungen vom Verfassungsgericht getroffen worden, darunter ca. 11.500 Grundrechtsentscheidungen, was einen Schnitt von 1.000 Entscheidungen pro Jahr bedeutet; davon sind $72 \%$ Grundrechtsentscheidungen und $28 \%$ Entscheidungen der abstrakten Normenkontrolle. Im ganzen Land sind ca. 1 Million verfassungsrechtliche Entscheidungen 
in den 17 Jahren seit der Verfassungsänderung gefällt worden; darunter betrafen ca. $70 \%$ den Schutz sozialer Grundrechte, wie die Rechte auf Existenzminimum, medizinische Versorgung, Bildung, Arbeit, auf Wohnung und auf soziale Sicherheit. Ca. $65 \%$ der Verfassungsbeschwerden bezüglich sozialer Grundrechte wurden positiv entschieden.

\section{Das Verfassungsgericht}

Dem kolumbianischen Verfassungsgericht ist den Schutz der Verfassung als höchstrangiger Norm anvertraut. Dazu soll das Gericht die Verfassungsnormen sowie die Normen internationaler Menschenrechtsschutzabkommen -z.B. die Internationalen Menschenrechtspakte der UNO oder die Amerikanische Menschenrechtskonvention- anwenden, da die ratifizierten Internationalen Verträge Verfassungsrang besitzen.

\section{Zusammensetzung und Amtsperiode}

Das Verfassungsgericht besteht aus neun Richtern mit einer Amtsperiode von einmalig acht Jahren. Die Mitglieder werden vom Senat als Zweiter Kammer des Nationalkongresses gewählt, wobei der Staatspräsident, das Oberste Gericht ("Corte Suprema de Justicia") und der Oberste Verwaltungsgerichtshof das ausschließliche Vorschlagsrecht haben.

\section{Entscheidungsarten und -effekte}

Während (abstrakte) Normenkontroll-Entscheidungen erga omnes wirken, gelten Grundrechtsentscheidungen inter partes. Bevor sich Kolumbien mit der Verfassungsänderung 1991 anstelle des bisherigen europäisch-kontinentalen Systems für das angloamerikanischen Präzedenzfall-System entschied, hatte seine Grundrechtsrechtsprechung keine bedeutsame Reichweite. Dieses zu eng konzipierte Grundrechtsschutzsystem ist nunmehr durch die Rechtsprechung des Verfassungsgerichts ausgedehnt. Das Verfassungsgericht verleiht über das Gesetz hinausgehend (ultra legem) seinen Entscheidungen entweder ,inter partes“ Wirksamkeit (jeder, der sich in einer identischen Situation befindet, kann sich auf die Entscheidung berufen, z.B. alle Arbeiter einer Firma sind in ihren Grundrechten verletzt worden) oder „,inter communis“ Wirksamkeit (jeder, der sich in einer identischen abstrakten Situation befindet, kann sich auf die Entscheidung berufen; z.B. alle Arbeiter, die generell in ihren Grundrechten verletzt wurden). Das kolumbianische Verfassungsgericht hat zudem die Doktrin „des allgemeinen verfassungswidrigen Zustands“ (,estado de cosas inconstitucional") entwickelt, wodurch die Verfassungsrichter verschiedene generelle Befehle an Behörden erteilen können, um eine massive Verletzung der Grundrechte mehrerer sich in gleicher Situation befindlicher Personen zu vermeiden oder zu beenden. Diese Doktrin wurde vom Verfassungsgericht zum Beispiel in Fällen verwendet, in welchen die Grund- 
rechte von Verhafteten ${ }^{2}$, Vertriebenen ${ }^{3}$ oder Patienten ${ }^{4}$ durch ein strukturelles staatliches Unterlassen verletzt wurden oder hätten verletzt werden könnten.

\section{Leitlinien der verfassungsrechtlichen Rechtsprechung bezüglich sozialer Grundrechte}

Einige Leitlinien der verfassungsgerichtlichen Rechtsprechung zu sozialen Grundrechten sollen kurz dargestellt werden. Sie lassen sich in sechs Punkten zusammenfassen:

\section{Minimalismus}

Die Verfassungs-Rechtsprechung zum Existenzminimum in Kolumbien schützt einen Kerngehalt sozialer Rechte: Nahrung, Obdach, medizinische Grundversorgung, schulische Grundbildung und soziale Hilfe ${ }^{5}$. Das Verfassungsgericht entscheidet, was bei der wirtschaftlichen und sozialen Sicherung des Einzelnen geboten, verboten oder ansonsten erlaubt ist. Letzteres steht im Ermessen politischer Entscheidungsträger. Was indes verfassungsrechtlich geboten oder verboten ist, gehört zum Bereich der Grundrechte und ist durch verfassungsrechtliche Verfahren zu sichern. Zum Beispiel hat das Verfassungsgericht eine Steuerreform, welche die Vereinheitlichung und die Ausdehnung der Mehrwertsteuer auf alle Produkte und Leistungen beabsichtigte, für verfassungswidrig erklärt, weil sie das Recht der Bevölkerung auf ein Existenzminimum verletzte ${ }^{6}$.

Das Recht auf Existenzminimum dient dem verfassungsrechtlichen Schutz minimaler Grundbedürfnisse des Einzelnen, soweit dieser bis zum Abschluss des instanzgerichtlichen Verfahrens nicht warten kann. Das gilt insbesondere bei Lohn- und Rentenforderungen, die nicht rechtzeitig vom Arbeitsgeber bezahlt werden ${ }^{7}$. Die Annerkennung des Rechts auf Existenzminimum sichert allerdings eine Person nicht vor jedwedem sozialen Risiko; sie vermeidet nur, dass jemand unter das materielle Niveau sinkt, welches es möglich macht, ihn als gleichwertig mit allen anderen anzuerkennen.

\section{Internationalisierung}

Mit Blick auf die Internationalisierung der Menschenrechtsstandards beim Schutz von sozialen Grundrechten bezieht sich das kolumbianische Verfassungsgericht regelmäßig auf die Leitlinien des Interamerikanischen Gerichtshofes für Menschenrechte, des ECOSOC

Corte Constitucional, Sentencia T-153 de 1998.

3 Corte Constitucional, Sentencia T-025 de 2004.

4 Corte Constitucional, Sentencia T-760 de 2008.

5 Rodolfo Arango / Julieta Lemaitre,. Jurisprudencia constitucional sobre el derecho al mínimo vital, Bogotá 2002, pp. 1-129.

6 Corte Constitucional, Sentencia C-776 de 2003.

7 Corte Constitucional, Sentencia T-148 de 2002.
} 
oder der ILO. In diesem Zusammenhang hat das Verfassungsgericht ein ministerielles Streikverbot für Staatsbedienstete für verfassungswidrig erklärt, weil nach ILO-Normen eine unabhängige Stelle und nicht die Regierung die Legalität eines Streiks zu überprüfen hat. ${ }^{8}$

Als Gewinn für die Förderung sozialer Grundrechte erweist sich die Zusammenarbeit zwischen Gewerkschaften und Menschenrechtsorganisationen. Um dem Abbau gesetzlicher Arbeitsgarantien, einer Deregulierung des Arbeitsmarktes und unbegrenzter Privatisierung das Recht auf soziale Sicherheit entgegenzusetzen, nutzen Gewerkschaften und soziale Organisationen das internationale Menschenrechtsschutzsystem. Gewerkschaften und Nicht-Regierungs-Organisationen berufen sich auf internationale Menschenrechtsstandards im Bereich der Tarifverträge und der sozialen Sicherheit. Die Internationalisierung der Menschenrechte begünstigt ihre Konstitutionalisierung auf nationaler Ebene.

\section{Subsidiarität}

Der Aktivismus des Verfassungsgerichts zugunsten sozialer Grundrechte macht die ordentliche Gerichtsbarkeit (Familien-, Zivil-, Straf- oder Verwaltungsgerichte) keineswegs überflüssig. Das Subsidiaritätsprinzip steht dem entgegen. Weil die ordentlichen Gerichte über "einfaches" Gesetzesrecht entscheiden, wird durch die Verfassungsgerichtsbarkeit nur ein Kerngehalt sozialer Grundrechte geschützt. Die Forderungen außerhalb dieses verfassungsrechtlichen Kerngehalts müssen vor ordentlichen Gerichten eingeklagt werden.

Bei Entscheidungen über Verfassungsklagen zum Schutz der Grundrechte (acción de tutela) wendet das Verfassungsgericht kein einfaches Gesetzesrecht an, sondern beruft sich auf Verfassungsnormen, was verfassungswidrige Entscheidungen ausschließt. Im Gegensatz zur ordentlichen Gerichtsbarkeit, sind auf der Ebene der Verfassungsgerichtsbarkeit die Verfassungsgrundsätze ausschlaggebend. Nicht die strikte Anwendung des Gesetzes sondern die Maximierung der Grundrechtsnormen ist die Hauptfunktion des Verfassungsgerichts. Die Grundrechte legen minimale Gerechtigkeitsstandards fest; sie sollen durch die verfassungsgerichtliche Intervention gesichert werden, wenn die Gesetzesanwendungs- und Gesetzesauslegungsmöglichkeiten der Fachgerichtsbarkeit erschöpft sind.

\section{Solidarität}

Letzteres bedeutet aber nicht, dass in Kolumbien - ähnlich wie in anderen Ländern Lateinamerikas - eine Konstitutionalisierung des Rechts ausgeblieben wäre. Im Gegenteil, die umfangreiche Rechtssprechungspraxis des kolumbianischen Verfassungsgerichts zeigt, dass das Solidaritätsprinzip und das Prinzip der Integralität der Menschenrechte ernst genommen werden. Zum Beispiel haben die Verfassungsentscheidungen zum Schutz der Grundrechte der durch den inneren bewaffneten Konflikt Vertriebenen dazu geführt, dass der 
Staat mehrere Billionen Pesos zur Wahrung der minimalen internationalen Richtlinien zum Schutz der Menschenrechte von intern vertriebenen Personen bereitstellen musste ${ }^{9}$.

Die Verfassungsklage verhindert, dass die Grundrechte der Person bei der politischen Zielsetzung und der Durchführung der staatlichen Aufgaben zurücktreten müssen oder gar verletzt werden. Die subjektive Durchsetzungskraft der sozialen Grundrechte vermeidet, sie nur als politische Zielvorstellungen oder objektive Normen zu betrachten. Dass soziale Grundrechte allerdings nur graduell erfüllbar sind, bedeutet nicht, dass sie keine Effektivität besäßen. Das Solidaritätsprinzip schließt aus, den Einzelnen in Notsituationen allein zu lassen, wie dies beispielsweise im Fall der verfassungsrechtlichen Gewährleistung des Rechts auf ein Existenzminimum deutlich wird. Insoweit ist das Solidaritätsprinzip ein klares Bekenntnis zur Erfüllung der staatlichen sozialen Pflichten gegenüber Obdachlosen, Behinderten, Kranken oder Individuen in Notsituationen ${ }^{10}$.

\section{Differenzierung}

Um für benachteiligte Personen oder Gruppen den Schutz sozialer Grundrechte zu optimieren, bedient sich die Verfassungsrechtsprechung der Differenzierung zwischen den verschiedenen Bevölkerungsgruppen. Das belegt eine Entscheidung, die eine gesetzliche Gesundheitsreform für verfassungswidrig erklärte, weil sie die besonderen Bedürfnisse indigener Völker bei der Strukturierung des Gesundheitssystems nicht ausreichend berücksichtigt hatte ${ }^{11}$. Mit Hilfe des Differenzierungsprinzips hat das Verfassungsgericht eine rechtliche Kategorie ("personas o grupos dignos de protección especial ") entwickelt, nach der Personen oder Gruppen rechtlich besser als andere Gesellschaftsmitglieder behandelt oder gestellt werden sollen, wenn sie sich in einer extremen Notsituation befinden. So hat das Verfassungsgericht die Rechte auf Leben und körperliche Unversehrtheit von Personen in extremer Armut ${ }^{12}$ geschützt, die wegen ihrer materiellen Lage von staatlichen Förderungen ausgeschlossen waren. Nach Feststellung einer Verletzung des Rechts auf Chancengleichheit einer alten und armen Person, der die Verwaltung keine ausreichende und rechtzeitige Auskunft über den Zugang zur staatlichen Sozialhilfe gegeben hatte, ordnete das Verfassungsgericht die Aufnahme der Rechtsträgerin in das einschlägige Förderungsprogramm an. Als weitergehende Folge dieser fortschrittlichen Rechtsprechung hat das Verfassungsgericht die Aufgabe übernommen, die Realisierung des Sozialstaatsprinzips im Rahmen einer Verhältnismäßigkeitsprüfung des staatlichen Tuns oder Unterlassens zu überwachen $^{13}$.

Corte Constitucional, Sentencia T-025 de 2004.

Corte Constitucional, Sentencias T-1330 de 2001 y T-149 de 2002.

11

Corte Constitucional, Sentencia C-088 de 2001.

12

13

Corte Constitucional, Sentencias T-401 de 1992 y T-533 de 1992.

Corte Constitucional, Sentencia T-149 de 2002. 


\section{Ausstrahlungswirkung}

Infolge der Ausstrahlungswirkung des Sozialstaatsprinzips und der Grundrechtsnormen auf das ganze kolumbianische Rechtssystem sind in den letzten Jahren auch wichtige Entscheidungen im Bereich des Wohnungsfinanzierungsgesetzes oder des Gesundheitswesens getroffen worden. Im ersten Fall hat das Verfassungsgericht das langfristige Finanzierungssystem zum Wohnungserwerb für verfassungswidrig erklärt, weil die gesetzliche Zinsberechnungsform wegen ihrer Enteignungswirkung das Grundrecht auf Wohnung verletzte. In einer erst vor kurzem gefällten Entscheidung hat das Verfassungsgericht den Gesetzgeber aufgefordert, vorsorglich bis zu einem bestimmten Termin die Gesundheitsleistungen für Wohlhabende und Arme zu vereinheitlichen, damit diese Normen nicht für verfassungswidrig erklärt würden.

Dank einer derartig fortschrittlichen verfassungsgerichtlichen Grundrechtsinterpretation zugunsten sozial ausgeschlossener oder schlecht stehender Personen, die alle Bereiche des einfach gesetzlichen Rechts umfasst, nehmen Behörden und Private in Kolumbien nunmehr Grundrechte und Verfassungsgrundsätze im Rahmen einer systematischen Auslegung durchaus ernst. Obwohl das Verfassungsgericht durch seine Rechtsprechung über den Ausstrahlungseffekt der sozialen Grundrechte allein außerstande ist, die Probleme der Armut und der Ungleichheit zu lösen, erfüllt es doch eine wichtige pädagogische und kritische Funktion: Es legt die Richtlinien eines humanen inklusiven Rechtsverständnisses und seiner Anwendung fest.

\section{Schlussfolgerungen}

Die Rechtsvergleichung ist eine Form multikultureller Kommunikation. Die Verrechtlichung der Staatsbeziehungen und der globale Konstitutionalismus fordern einen ständigen, offenen aber kritischen Dialog zwischen den Kulturen. Das Beispiel, wie die Verfassungsgerichtsbarkeit Kolumbiens mit den sozialen Grundrechten umgeht, betrifft zentrale Fragen der Beziehung von Recht und Politik. Welches sind die Auswirkungen des verfassungsgerichtlichen Aktivismus auf das demokratische Staatsrechtssystem? Fördern die Verfassungsentscheidungen über soziale Grundrechte tatsächlich das Demokratieprinzip und die Menschenrechtsverwirklichung? Oder bedeutet die Anerkennung sozialer Rechte auf Verfassungsebene vielmehr die Zerstörung des demokratischen Rechtstaats und führt zu seiner Ersetzung durch einen Justizstaat, wie E.-W. Böckenförde meinte? Diese Fragen können hier nicht weiter erörtern werden ${ }^{14}$. Konkrete Beispiele, die beweisen, dass dieser nicht der

Siehe dazu Rodolfo Arango, Basic Social Rights, Constitutional Justice and Democracy, Ratio Juris 16-2 (2003), pp. 141-154; Rodolfo Arango, Realizing Constitutional Social Rights Through Judicial Protection, Beyond Law 25 (2002), pp. 71-86; Rodolfo Arango, Regionaler und internationaler Schutz sozialer Menschenrechte. Von der Philosophy zur Kodifizierung, in: Thomas Frank / Anne Jenichen / Nils Rosemann (eds.), Soziale Menschenrechte - die vergessenen Rechte? Zur Unteilbarkeit der Menschenrechte - ein interdisziplinärer Überblick, Berlin 2001, pp. 43-64; Rodolfo Arango, On Constitutional Social Rights, in: Burton Leiser / Tom D. Campbell (eds.), 
Fall ist, sind nützlich. Die oben erwähnten Entscheidungen des kolumbianischen Verfassungsgerichts zeichnen sich aus durch hohe Rationalität und beispielhafte Wirkungen. Trotzdem wird es bis zur Demokratisierung und Menschenrechtsverwirklichung in Lateinamerika noch dauern. Das hängt davon ab, welche Konzeption der Demokratie und der Menschenrechte verfolgt wird. Normalerweise bevorzugen entwickelte Länder eine Konzeption der liberalen, repräsentativen Demokratie, wobei zivilen und politischen Rechten normativ der Vorrang gegenüber wirtschaftlichen, sozialen und kulturellen Rechten eingeräumt wird. Obwohl diese Konzeption geschichtlich zu erklären ist, steht sie mit der heutigen Menschenrechtsauffassung ,interdependenter Rechte“ (Interdependenz-Prinzip) und „,vollständiger Rechte“ (Integralitäts-Prinzip) nicht in Einklang ${ }^{15}$. Die so genannten "nicht wohlgeordneten Gesellschaften" der so genannten "Dritten Welt" sind geprägt durch strukturellen Missbrauch öffentlicher Macht und extreme wirtschaftliche Ungleichheit bei zunehmend konfligierender sozialer Marginalisierung. Dagegen steht das Verfassungsverständnis einer sozialen und partizipatorischen Demokratiekonzeption, nach welcher keine wesentlichen Unterschiede zwischen zivilen und politischen Rechten einerseits und wirtschaftlichen, sozialen und kulturellen Rechten andererseits bestehen. Die kolumbianische verfassungsrechtliche Entwicklung weist in die Richtung, dass eine minimale soziale Gerechtigkeit gesichert werden soll - und muss -, damit eine Integration Lateinamerikas gelingt.

Human Rights in Philosophy and Practice (Applied Legal Philosophy). Ashgate 2001, pp. 141152.

Amartya Sen, Welfare Economics and Two Approaches to Rights, in: J. Casas / F. Schneider (eds.), Current Issues in Public Choice, Cheltenham / Brookfield 1996, pp. 21-39. 